\title{
Commentaries
}

\section{Genetic instability in colorectal cancer}

The development of neoplasia has been thought to be associated with genetic alterations for many years. In colorectal cancer two specific types of genetic instability have been defined. One is associated with chromosomal instability and the other with DNA microsatellite instability, also known as replication errors. ${ }^{1}$ The mechanism underlying tumorigenesis leads to profound differences in the pathological features, prognosis and response to chemotherapy of these two types of colorectal cancer.

The great majority of colorectal cancers are aneuploid with a variable chromosome complement. However, between 10 and $15 \%$ of colorectal cancers are near diploid and exhibit DNA microsatellite instability in which replication errors may be demonstrated within repetitive sequences of tumour DNA which are not present in the normal DNA of the individual. Tumours with microsatellite instability occur more frequently in younger patients, are more frequently proximal to the splenic flexure and exhibit exophytic growth, poor differentiation, extracellular mucin production, and a Crohn's-like lymphocytic reaction. ${ }^{2}$ The genes mutated during tumorigenesis may be different from those in aneuploid tumours with frequent mutations of tumour growth factor $\beta$ receptor II and Bax. ${ }^{34}$ Cell lines from these tumours are less sensitive to alkylating agents such as N-methyl-N'-nitro-N-nitrosoguanidine (MNNG). ${ }^{5}$

Hereditary non-polyposis colon cancer (HNPCC) is an autosomal dominant predisposition to colorectal neoplasia. It has been defined clinically by the Amsterdam criteria of the International Collaborative Group for HNPCC as colorectal cancer affecting at least three members of a family, one of whom must be a first degree relative of the other two, with at least two successive generations affected and one case diagnosed under the age of $50 .{ }^{6}$ Colorectal carcinomas in HNPCC exhibit microsatellite instability and share the same pathological characteristics.

In HNPCC the microsatellite instability is due to germline mutations of DNA mismatch repair genes. These genes were identified because a similar type of DNA instability had been described in yeast. Mutations of the human homologues of the yeast genes segregate with disease in HNPCC families.

Brown et al, in this issue (see page 553), have looked at the proportion of cases of familial colorectal cancer associated with microsatellite instability and which, by implication, may be due to the same DNA mismatch repair gene mutations that cause HNPCC. They identified two groups of patients with colorectal cancer. The first group was from follow up of one surgeon's clinical practice and a careful family history had been taken. The second group was from a regional genetic clinic and the families fulfilled the Amsterdam criteria for HNPCC. In the follow up population only $4 / 479(0.8 \%)$ had a family history that fulfilled that Amsterdam criteria and only one of these had a tumour that showed microsatellite instability. In the families from the regional genetic centre $8 / 16$ had tumours with microsatellite instability. In four of these families a germline DNA mismatch repair gene mutation had been identified and all of these families had tumours which exhibited microsatellite instability.

These results suggest that only a small proportion of familial colorectal cancer, and possibly only $50 \%$ of families fulfilling the Amsterdam criteria for HNPCC, is associated with microsatellite instability. This indicates that there are further inherited predispositions to colorectal cancer yet to be identified.

The majority of colorectal cancers are aneuploid with a variable chromosome number. Genetic alterations involving the loss of chromosomes that occur in tumorigenesis may be demonstrated by comparing normal and tumour DNA from the same individual. Loss of constitutional heterozygosity (allele loss) may indicate areas where tumour suppressor genes have been inactivated. This technique has been used to identify many of the genes which are mutated in colorectal tumorigenesis.

To date, it has not been clear whether aneuploidy is an important mechanism or a by-product of tumorigenesis. However, recent work from Vogelstein's group indicates that aneuploidy may be significant in pathogenesis. ${ }^{7}$ A gene that causes aneuploidy in colorectal cancer has been described. ${ }^{8}$ This is the human homologue of BUBI a yeast gene that is critical in the control of the formation of the mitotic spindle. Mutations of hBUBI are seen in some aneuploid human colorectal cancers. The functional role of hBUBI has been demonstrated by the transfer of the mutant gene to diploid human colon cancer cell lines leading to the development of aneuploidy.

In conclusion, two different genetic pathways may lead to colorectal tumorigenesis due to either chromosomal or DNA microsatellite instability. The molecular basis of these two types of carcinoma have been defined and the two types of tumours shown to have very different biological behaviour. In familial colorectal cancer Brown et al have shown that only a small proportion of cancers have the microsatellite instability characteristic of HNPCC and that there are likely to be other genetic causes.

ICRF Colorectal Unit,

H J W THOMAS

St Mark's Hospital,

Watford Road,

Middlesex HA1 3UF, UK

1 Kinzler KW, Vogelstein B. Lessons from hereditary colorectal cancer. Cell 1996;87:159-70.

$2 \mathrm{Kim} \mathrm{H}$, Jen J, Vogelstein B, et al. Clinical and pathological characteristics of sporadic colorectal carcinomas with DNA replication errors in microsatellite sequences. Am f Pathol 1994;145:148-56.

3 Rampino N, Yamamoto H, Ionov Y, et al. Somatic frameshift mutations in the BAX gene in colon cancers of the microsatellite mutator phenotype. Science 1997;275:967-9.

4 Markowitz S, Wang J, Myeroff L, et al. Inactivation of type II TGF- $\beta$ receptor in colon cancers cells with microsatellite instability. Science 1995;268: 1336-8.

5 Carethers JM, Hawn MT, Chauhan DP, et al. Competency in mismatch repair prohibits clonal expansion of cancer cells treated with N-methyl-N'nitro-N-nitrosoguanidine. F Clin Invest 1996;98:199-206.

6 Vasen HF, Mecklin JP, Khan PM, et al. The International Collaborative Group on Hereditary Non-polyposis Colorectal Cancer (ICG-HNPCC). Dis Colon Rectum 1991;34:424-5.

7 Lengauer C, Kinzler KW, Vogelstein B. Genetic instability in colorectal cancers. Nature 1997;386:623-7.

8 Cahill DP, Lengauer C, Yu J, et al. Mutations of mitotic checkpoint genes in human cancers. Nature 1998;392:300-3. 


\section{Appendicectomy and childhood hygiene: different sides of the same coin?}

The aetiology of ulcerative colitis and Crohn's disease remains an enigma. There is, however, a growing consensus that early events/exposure are of importance and constitute a "window of opportunity". Both analytical and observational studies as well as animal models give credence to this hypothesis. Smoking has, until recently, been the only factor that has been consistently associated with an increased risk of Crohn's disease and a decreased risk of ulcerative colitis. Appendicectomy has now been added to this very short list as there has been a consistent finding in several studies that this operation is associated with a decreased risk of ulcerative colitis. ${ }^{12}$ Duggan et al's study (see page 494) confirms these results. Their findings for childhood hygiene are in accordance with previous studies where the availability of hot running water is associated with Crohn's disease but not ulcerative colitis. ${ }^{3}$ However, no other aspects of childhood hygiene were associated with either Crohn's disease or ulcerative colitis, including Helicobacter pylori serology. In the case of appendicectomy, their results are also in accordance with previous studies, showing that appendicectomy is associated with a strong protective effect against ulcerative colitis $^{12}$ which is even more pronounced if surgery was performed before the age of 20 years.

\section{How should the findings be interpreted?}

Duggan et al are extremely cautious when interpreting their results especially with regard to hot water supply in childhood, but underline that their findings constitute further evidence that exposure to certain environmental factors during childhood is important. However, with regard to appendicectomy the authors emphasise that removal of the appendix could be causally linked to the decreased risk of ulcerative colitis. This emphasis is supported by results in an animal model $^{4}$ but an alternative explanation, not necessarily mutually exclusive, is that appendicitis could be inversely associated with the risk of ulcerative colitis. In Sweden there has been a dramatic decline, from 13000 to 10000 operations annually, in the number of appendicectomies performed over the past 10 years. This decline is most pronounced in younger age groups. ${ }^{5}$ Moreover, there are strong indications that this effect is not due to better diagnostic procedures but actually reflects a decrease in the incidence of appendicitis. The underlying reason for this decline is unclear, but unspecified childhood exposure could also be relevant to the lifetime risk of appendicitis as illustrated by the hypothesis put forward by Barker ..."That the rise of appendicitis in western Europe since the late 19 th century could be due to improvement in sewage disposal and water supplies, leading to an enteric infection in childhood at an older age." ${ }^{\prime 6}$ The increased use of day care centres for very young children since the late 1960s could be the underlying reason for the decreasing trend.

Previous observational studies have shown an association between both ulcerative colitis and Crohn's disease and early infectious events. ${ }^{78}$ Moreover, in studies from the 1960 s and 1970s early weaning was implicated as a risk factor for both diseases and a similar finding has been reported for high socio-economic status. ${ }^{89}$ Later studies have not confirmed these associations. ${ }^{27}$ The assumption that hot water supply during the first part of this century was crucial for the better survival of young children with severe infections, which were more likely to occur after early weaning, could explain Duggan et al's findings. In later studies the absence of an association with both socioeconomic status and early weaning outside the United Kingdom could be explained by better hygiene and a decrease in infant mortality. The two diverging trends of an increase in the incidence of ulcerative colitis and Crohn's disease and a decrease in that of appendicitis could therefore be different sides of the same coin.

One way of resolving this issue would be to examine the need for appendicectomy in a larger data set. Such studies should focus on the extent to which there is a difference in the protective effect against ulcerative colitis among those undergoing appendicectomy for appendicitis compared with those subsequently found to have a normal appendix at surgery. The results from such studies should reveal whether appendicectomy alone protects against ulcerative colitis or whether appendicitis is necessary.

Department of Epidemiology,

A EKBOM

University Hospital,

S-751 85 Uppsala, Sweden

1 Rutgeerts P, D’Haens G, Hiele M, et al. Appendectomy protects against ulcerative colitis. Gastroenterology 1994;106:1251-3.

2 Gilat T, Hacohen D, Lilos P, et al. Childhood factors in ulcerative colitis and Crohn's disease. Scand f Gastroenterol 1987;22:1009-24.

3 Gent AE, Hellier MD, Grace RH, et al. Inflammatory bowel disease and domestic hygiene in infancy. Lancet 1994;343:766-7.

4 Mizoguchi A, Mizoguchi E, Chiba C, et al. Role of appendix in the development of inflammatory bowel disease in TCR- $\alpha$ mutant mice. $\mathcal{F}$ Exp Med 1996;184:707-15.

5 Blomqvist P, Ljung H, Ekbom A, et al. Appendectomy in Sweden 1989-1993. Assessed by the Inpatient Register. F Clin Epidemiol 1998: in press.

6 Barker DJP, Morris JA. Acute appendicitis, bathrooms, and diet in Britain and Ireland. BMf 1988;296:953-8.

7 Ekbom A, Adami HO, Helmick C, et al. Perinatal risk factors for inflammatory bowel disease: a case-control study. Am f Epidemiol 1990;132:111119 .

8 Whorwell PJ, Holdstock G, Whorwell GM, et al. Bottle feeding, early gastroenteritis and inflammatory bowel disease. $B M \mathcal{F}$ 1979;1:382.

9 Acheson ED, Nefzger MD. Ulcerative colitis in the United States Army in 1944. Epidemiology: comparisons between patients and controls. Gastroenterology 1963;44:7-19.

\section{AFAP: variety is the spice of life}

Familial adenomatous polyposis (FAP) has been known for over 100 years ${ }^{1}$; Gardner's syndrome, the familial association of multiple colonic adenomas and early onset colorectal cancer (CRC) with osteomas and multiple cutaneous fibromas or epidermoid cysts has been known for almost 50 years. $^{2}$ The discovery of the APC gene ${ }^{3}$ has changed the diagnosis and clinical management of FAP radically. Firstly, it became clear that FAP and Gardner's syndrome have the same genetic basis. The expression of osteomas and of the cutaneous signs varies greatly among people with the same mutation. Other phenotypic variations have been found to have a genetic basis. For example, congenital hypertrophy of the retinal pigment epithelium is associ- 
ated with mutations distal to exon $9,{ }^{4}$ whereas susceptibility to desmoid tumours is associated with mutations at codons 1444 and $1578^{5}$ and a profuse number of colonic adenomas, often more than 5000 (compared with the more typical 1000-2000), is associated with mutations between codons 1250 and $1464 .^{6}$

A variant called attenuated FAP (AFAP) has been associated with mutations in the 5 ' portion of the APC gene. ${ }^{7}$ AFAP is characterised by the occurrence of often fewer than 100 colonic adenomas (sometimes as few as five or 10), with proximal colonic predominance, a later age of adenoma development (c. 35-40 years), and a still later age of CRC, often in the mid-50s. These features require screening by colonoscopy rather than flexible sigmoidoscopy. Given the later age of colonic adenoma and CRC onset, we initiate colonoscopy at age 20-25 years. This recommendation would be modified by the occurrence of earlier colonic neoplasia in the family. Unlike classic FAP, there is no age where surveillance can be discontinued on account of a normal phenotype. The adenomas are also often flat. Fundic gland polyps and duodenal adenomas may also occur, hence the need for upper endoscopy with a wide angle endoscope for all patients with FAP regardless of location of mutation.

Brensinger and colleagues (see page 548) have studied 31 at-risk or affected members from four families with a mutation in the opposite end of the APC gene (codon 1979 or 2644). The phenotype was remarkably similar to AFAP. Among mutation carriers, some examined at an older age had fewer than 100 colorectal adenomas whereas other family members may have presented with classic (profuse) polyposis at a young age. CRC occurred at a mean age of 50 years, which was much older than that found in classic FAP pedigrees. Extracolonic phenotypic findings known to associate with classic FAP were variably expressed in these families. These authors reported a disassociation of severity of extracolonic manifestations from the number of colorectal polyps. We have observed the same thing in AFAP. In our limited experience, we have observed fundic gland polyps, duodenal adenomas and periampullary carcinoma, but there seems to be a paucity of other extracolonic manifestations relative to classic FAP. However, more research is needed on this topic.

The diagnostician must be aware of the phenotypic importance of mutation location within the APC gene. However, do we know enough about these phenotypic correlations to use this information for genetic counselling and, ultimately, for surveillance and management of these patients at high risk of cancer? Are these phenotypic correlations clinically important enough to say that all patients with FAP should be tested to identify the mutation? We believe so. There is no question that colonoscopy, as opposed to flexible sigmoidoscopy, is the favoured screening approach in AFAP (given its proximal predilection to colonic adenomas) or in any extreme $3^{\prime}$ or $5^{\prime}$ mutation in APC. When too many colonic adenomas are present for polypectomy, particularly when they are clustered in the caecum, we have recommended prophylactic subtotal colectomy. However, in cases with small numbers of adenomas, management by polypectomy is an option. Having said this, the efficacy of polypectomy to prevent CRC in these patients has not been established.

Because of the late onset of both colonic adenomas and CRC, its right-sided adenoma predilection, and the fact that the colonic adenomas may be so few $(<10)$, the importance of AFAP may be completely missed unless a comprehensive family history is obtained and carefully examined. This history must account for colonic adenomas, their age of onset, location within the colon, and the occurrence of CRC. Indeed, there is a dire need for education of physicians about AFAP, with particular attention given to how to diagnose and manage it, since in our experience, in spite of the fact that the disorder was described a decade ago, ${ }^{89}$ a surprisingly small number of physicians express knowledge of the syndrome and/or confidence in its diagnosis and management.

When do we order a test on a young patient with multiple colonic adenomas and a negative or unremarkable family history, and how do we interpret such test results? This is a vexing problem for which the answer remains elusive. We recommend APC gene testing for any patient with more than five colonic adenomas at an early age $(<25$ years). Unfortunately, a negative molecular genetic test for mutation in the APC gene will not rule out a genetic cancer risk to the patient, siblings or progeny. He or she might harbour a germline mutation that has not yet been identified. Roughly $20 \%$ of frank FAP cases have negative mutation tests. ${ }^{10}$ The patient's early adenomas could be a manifestation of another hereditary CRC syndrome such as hereditary non-polyposis colorectal cancer (HNPCC). Conversely, should an APC mutation or, for that matter, a mutation for other hereditary forms of CRC such as HNPCC, be identified, then one would be in a position to do DNA testing on that individual's parents, siblings, and progeny. This may be especially valuable in AFAP because of the absence of a florid phenotype in many cases.

No solid data exist on the true population frequency of AFAP. Such estimates will be difficult, due to its subtle phenotypic manifestations coupled with the fact that the detailed family histories necessary for the diagnosis of AFAP are notoriously ignored in the clinical practice setting.

H T LYNCH
P WATSON
Department of Preventive Medicine and Public Health,
Creighton University School of Medicine,
2500 California Plaza,
Omaha, Nebraska 68178, USA

1 Bulow S. Familial polyposis coli. Dan Med Bull 1987;34:1-15

2 Gardner EJ, Richards RC. Multiple cutaneous and subcutaneous lesions occurring simultaneously with hereditary polyposis and osteomatosis. $\mathrm{Am} \mathcal{F}$ Hum Genet 1953;5:139-47.

3 Kinzler KW, Nilbert MC, Su L-K, et al. Identification of FAP locus genes from chromosome 5q21. Science 1991;253:661-5.

4 Olschwang S, Tiret A, Laurent-Puig P, et al. Restriction of ocular fundus lesions to a specific subgroup of APC mutations in adenomatous polyposis coli patients. Cell 1993;75:959-68.

5 Caspari R, Olschwang S, Friedl W, et al. Familial adenomatous polyposis: desmoid tumours and lack of ophthalmic lesions (CHRPE) associated with APC mutations beyond codon 1444. Hum Mol Genet 1995;4:337-40.

6 Nagase H, Miyoshi Y, Horii A, et al. Correlation between the location of germ-line mutations in the APC gene and the number of colorectal polyps in familial adenomatous polyposis patients. Cancer Res 1992;52:4055-7.

7 Spirio L, Otterud B, Stauffer D, et al. Linkage of a variant or attenuated form of adenomatous polyposis coli to the adenomatous polyposis coli (APC) locus. Am F Hum Genet 1992;51:92-100.

8 Lynch HT, Smyrk T, Lanspa S, et al. Flat adenomas in a colon cancer-prone kindred. F Natl Cancer Inst 1988;80:278-81.

9 Lynch HT, Smyrk T, McGinn T, et al. Attenuated familial adenomatous polyposis (AFAP): a phenotypically and genotypically distinctive variant of FAP. Cancer 1995;76:2427-33.

10 Powell SM, Petersen GM, Krush AJ, et al. Molecular diagnosis of familial adenomatous polyposis. $N$ Engl f Med 1993;329:1982-7. 


\section{Swallowing is a precarious business}

The upper oesophageal sphincter (UOS) remains closed at rest as a result of a combination of intrinsic muscle tone and passive pressure from the anterior mass of the larynx. This tonic activity is augmented during inspiration, thus preventing oesophageal air penetration each time intrathoracic pressure falls. UOS tonic pressure undergoes a further augmentation if the tubular oesophagus is distended-a protective reflex against oesophagopharyngeal regurgitation. Once oral preparation of an ingested bolus is complete, UOS tonic activity is abolished during the pharyngeal phase of a swallow. As the laryngopharynx elevates and shortens to engulf the bolus, the simultaneous forward tug of the rising hyoid on the anterior UOS wall brings about mechanical opening of the sphincter.

The nature of the deglutition reflex remains somewhat mysterious. The trigger to the onset of the ultra-rapid pharyngeal phase of swallowing is only semireflexive. All of us at times select the preferred moment of swallow-when we feel the bolus has been sufficiently chewed, when the social moment is right. Equally all of us have been caught out by the premature onset of a swallow reflex-when the bolus still feels too big to be swallowed, but seems to be escaping precipitately from the oropharynx. The elements of the swallow "reflex" which have been demonstrated experimentally to be under volitional control include the force of tongue and pharyngeal contraction, ${ }^{1}$ the duration of hyoid elevation ${ }^{2}$ and the extent of UOS opening. ${ }^{3}$

Until recently, the anterior pillars of the fauces were believed to be the site for afferent stimulation of the pharyngeal swallow reflex. The anterior pillars were subjected to "sensitisation" by application of cold laryngeal mirrors, in (almost certainly fruitless) attempts to facilitate the swallow reflex. The Chicago group, however, showed that prior to swallowing, the bolus collected on the tongue base - that is, in the oropharynx, distal to the oral cavity and the anterior faucial pillars. ${ }^{4}$ Moreover, it has been shown that a bolus of food reaches the vallecula prior to swallow onset in up to $50 \%$ of swallows. ${ }^{56}$

The epiglottic edge seems to be the most sensitive trigger zone for swallowing ${ }^{6}$ and is innervated by the vagus. Magnetic stimulation of either the trigeminal or vagal nerve evokes pharyngo-oesophageal EMG responses. Also, muscle responses to cortical stimulation are facilitated by vagal stimulation. ${ }^{7}$ Such modification of central swallow reflexes represents one method by which deglutition dynamics in the pharyngo-oesophageal segment adapt to alterations in bolus properties - for example, by enhanced and earlier UOS opening with increasing bolus volumes.

Swallowing is a precarious business because of the common upper channel for ingestion and respiration. A major function of the larynx is sphincteric - to protect the airway at moments of uncoordinated swallowing and respiration. The upper airway is normally protected from laryngeal penetration by deglutition apnoea, even in the minority of normal swallows which occur in inspiration.

A few years ago, the group in Wisconsin characterised, first in animals and later in humans, a number of reflexes which modify the normal resting and deglutitive patterns of pharyngo-oesophageal motility, most of which seem to enhance airway protection. These include the oesophagoglottal closure reflex, a protective closure of the upper airway introitus in response to abrupt oesophageal distension. This reflex is likely to protect the airway at times of notable gastro-oesophageal reflux, when there is a risk of oesophagopharyngeal reflux and laryngeal acid penetration. The reflex may be supported by a second laryngeal protective phenomenon, a pharyngoglottal adduction reflex, which is triggered by pharyngeal entry of very small amounts of fluid, especially in younger people. ${ }^{8}$

The group, lead by Reza Shaker, have also described a pharyngo-UOS contractile reflex where the stimulation of glossopharyngeal afferents by hypopharyngeal fluid evokes a vagal efferent tonic response in the UOS. Larger fluid volumes evoke a reflex pharyngeal swallow. These responses, which are abolished by topical pharyngeal anaesthesia, account for the difficulty encountered in early attempts at pharyngeal manometry with perfused catheter systems. In healthy volunteers the threshold volume for UOS contraction is only $0.1 \mathrm{ml}$ if infused rapidly, but around $1 \mathrm{ml}$ with slow infusion.

It is suggested that the UOS contraction response to the presence of extremely small amounts of fluid protects against aspiration. This protection may be brought about by three mechanisms. Firstly, the UOS tonic pressure augmentation reduces the risk of further oesophagopharyngeal reflux. Secondly, if fluid enters the proximal pharynx, as in the experimental situation, the UOS contraction may assist a sufficient fluid build up in the pharynx to provoke the related pharyngeal swallow, which clears the pharynx and also closes the glottis. Finally, Shaker's work on glottic closure during deglutition suggests that the pharyngo-UOS contractile reflex is accompanied by closure of the laryngeal inlet.

There seems to be little direct observation of the respiratory and other motor effects, if any, of fluid entering the pharynx as a result of oesophagopharyngeal reflux, one of the proposed aetiological factors in so called acid laryngitis. Shaker's team now report the effects of cigarette smoking on the UOS contractile and swallow reflex which follow experimental pharyngeal water infusion (see page 537). Smoking increased the threshold of the pharyngeal reflexes. This report also indicates an absence of the pharyngo-UOS contractile reflex in three of the 20 subjects. This may reflect the natural variation in pharyngeal reflexes (e.g. the gag reflex).

The most direct explanation for the group's findings is that cigarette smoke acts as a sensory depressant in the upper aerodigestive tract. Olfaction and taste are diminished in smokers, whose oropharyngeal mucosa is chronically thickened, as evinced by the reduced rate of aphthous ulceration in smokers. This argument does not, however, take account of the known abolition of the reflexes after topical anaesthesia, nor does it explain the acute incremental threshold rise after smoking. The authors examine a number of other mechanisms, ${ }^{9}$ but do not consider the role of nicotine induced arousal ${ }^{10}$ on pharyngeal reflex activity.

Shaker's team has made unique contributions to the understanding of the pathophysiology of pharyngolaryngeal coordination. Nonetheless, their latest paper should not let us lose sight of the fact that the principal effects of cigarette smoking on the respiratory tract are much more severe, and direct, than merely the diminution of pharyngeal fluid responsiveness.

Department of Otolaryngology Head and Neck Surgery,

J A WILSON

University of Nervcastle, Freeman Hospital,

Newcastle upon Tyne NE7 7DN, UK 
1 Pouderoux P, Kahrilas PJ. Deglutitive tongue force modulation by volition, volume and viscosity in humans. Gastroenterology 1995;108:1418-26.

2 Logemann JA, Pauolski BR, Rademaker AW, et al. Super-supraglottic swalow in irradiated head and neck cancer patients. Head Neck 1997;19:53540.

3 Kahrilas PJ, Logemann JA, Krugler C, et al. Volitional augmentation of upper oesophageal sphincter opening during swallowing. Am f Physio 1991;260:G450-6.

4 Ergun GA, Kahrilas PJ, Lin S, et al. Shape, volume and content of the deglutitive pharyngeal chamber imaged by ultrafast computerised tomography. Gastroenterology. 1993;105:1396-403.

5 Palmer JB, Rudin NJ, Lara G, et al. Coordination of mastication and swallowing. Dysphagia 1992;7:187-200.
6 Dua KS, Ren J, Bardan E, et al. Coordination of deglutitive glottal function and pharyngeal bolus transit during normal eating. Gastroenterology 1997;112:73-83.

7 Hamdy S, Aziz Q, Rothwell JC, et al. Cranial nerve modulation of human cortical swallowing pathways. Am f Physiol 1997;272:G802-8.

8 Shaker R, Lang IM. Reflex mediated airway protective mechanisms against retrograde aspiration. Am f Med 1997;103:64-73S.

9 Scott AM, Kellow JE, Shuter B, et al. Effect of cigarette smoking on solid and liquid intragastric distribution and gastric emptying. Gastroenterology 1996;104:410-16.

10 O'Neill ST, Parrott AC. Stress and arousal in sedative and stimulant cigarette smokers. Psychopharmacology 1992;107:442-6.

\section{Cholecystokinin infusion: assessing a rather provocative test}

All gastroenterologists, whether physicians or surgeons, will recognise a group of patients who have typical gall bladder pain but in whom imaging of the biliary tree remains obstinately negative. Incalculable numbers of acalculous patients, many of them middle-aged women, end up having a cholecystectomy almost by default. The results are surprisingly successful, perhaps owing to the powerful placebo effect of a surgical operation. Thus "blind" cholecystectomy relieved the pain in up to $70 \%$ of such patients in two historical series as opposed to the $80-85 \%$ that could be anticipated in those with symptomatic gallstones. ${ }^{12}$ The source of pain in such patients may lie within the biliary tract even if it cannot be clearly recognised. Chronic acalculous cholecystitis is the commonest suggested cause, but its definition varies between reports and is often not stated. Hyperplastic cholecytoses such as cholesterosis and adenomyosis (diverticulosis) may cause symptoms that are improved by cholecystectomy even in the absence of concomitant stones. ${ }^{3}$ The so called "cystic duct syndrome" is thought to result from postinflammatory stenosis or congenital tortuosity of the cystic duct and might cause right hypochondrical pain by inhibiting normal gall bladder emptying. In those without any clear pathological diagnosis, there could be a painful dysmotility due to asynchronous contraction of the neck and fundus of the gall bladder. All of these conditions would logically be improved by resection of the gall bladder, whereas common extrabiliary causes of pain such as irritable bowel syndrome should not be improved.

As preoperative diagnosis of acalculous disease of the gall bladder is difficult and the response to cholecystectomy is unpredictable, the cholecystokinin (CCK) provocation test has been advocated as a means of identifying patients who will benefit from the operation. There are two components to the test, but not all investigations have clarified the exact criteria for a positive result. The habitual pain should be reproduced during stimulation of the gall bladder muscle by an intravenous infusion of CCK, while measurement of gall bladder volume (for up to one hour) should demonstrate delayed emptying. Many authors have questioned the specificity and reproducibility of the pain response. ${ }^{4}$ CCK causes pain in some healthy individuals especially if the injection is rapid. It stimulates the smooth muscle of the small and large intestine and the sphincter of Oddi as well as the gall bladder, and these effects could lead to false positive results in those with intestinal motility disorders or sphincter dyskinesia. Ideally CCK studies should be placebo controlled and double blind, with the imaging and histopathology performed by independent observers.

In this issue (see page 571) Smythe and colleagues have investigated 58 patients with acalculous biliary pain by means of a CCK provocation test using serial ultrasound scans to determine the ejection fraction of the gall bladder. This study had the advantages of being prospective and of comparing the symptomatic response to operative and non-operative treatment in both CCK positive and CCK negative patients. After cholecystectomy, $67 \%$ of those with a positive test became symptom-free as opposed to $43 \%$ of those with a negative test; this difference was not statistically significant. With conservative management, $40 \%$ of those with a positive test lost their symptoms as opposed to $75 \%$ of those with a negative test, but numbers were small. When the test was repeated postoperatively, 20 of 25 patients converted from positive to negative, but interestingly the five who remained positive had persisting pain. Although the percentage reduction in gall bladder volume within an hour of CCK infusion was lower in positive than negative responders ( $33 v 52 \%$ ), this result did not correlate with the symptomatic benefit after cholecystectomy.

The authors conclude that cholecystectomy has a good chance of curing episodic right hypochondrical pain but that the CCK provocation test is of no predictive value. Their study can be criticised on four counts. Firstly, better prediction of symptomatic outcome could have been obtained if the operation had been performed on equal proportions of patients with positive and negative tests; the disproportion in the present study (84 v 54\%) will have introduced a verification bias to the statistical analysis. Secondly, although most of the resected gall bladders showed evidence of chronic cholecystitis on histological examination, this can be rather a subjective diagnosis and the criteria are not stated. It is not clear whether the pathologist examined the cystic duct for narrowing or identified any cases of cholecystosis. Thirdly, six months might be too early to assess postoperative pain relief. Fourthly, ultrasonography (or oral cholecystography) may not be the best way of measuring gall bladder emptying as the images are two dimensional and subject to considerable observer bias; a wide variation in ejection fractions (between 10 and 90\%) can be found among control subjects. ${ }^{5}$ CCK scintigraphy is probably more accurate, being three dimensional, computer based and operator independent. A maximal ejection fraction below 35\% correlates closely with the histological changes in the resected gall bladders (or cystic ducts) of those with acalculous biliary pain, and it can predict the symptomatic response to operation in such patients. ${ }^{67}$

The disappointing results of the present study on the role of the CCK provocation test support those of a previous study of 48 patients in which a positive clinical or radiological response to $\mathrm{CCK}$ could not predict the 
response to cholecystectomy. ${ }^{8}$ By contrast, a much larger study from Rhodes and colleagues showed that a positive CCK test had an excellent chance of predicting symptomatic benefit. Of 90 patients with pain after CCK infusion, $67 \%$ had complete resolution of symptoms postoperatively and another $24 \%$ had a noticeable improvement in symptoms. ${ }^{9}$

In conclusion, a case can still be made for performing CCK provocation studies in patients with biliary pain but a normal gall bladder on conventional imaging. At least two in three of those with a positive test will be helped by cholecystectomy. Although a negative test does not rule out the chance of a good postoperative result, it might encourage a further search for alternative diagnoses. Should that fail, patients may be offered an operation on the understanding that they have only a 50:50 chance of symptomatic relief. Pragmatists may feel that the limitations of the test are such that any fit patient with persisting symptoms should be advised to undergo cholecystectomy, particularly nowadays when minimal access techniques have reduced the metabolic "insult" of this procedure.
D AL-MUSAWI

Department of Gastrointestinal Surgery,

Imperial College School of Medicine

at the Hammersmith Hospital

Du Cane Road, London W12 ONN, UK

Correspondence to: Professor RCN Williamson.

1 Mackey WA. Cholecystitis without gallstones. Br 7 Surg 1934;22:274-95. 2 Gunn A, Keddie NC, Fox H. Acalculous gallbladder disease. $\mathrm{Br} \mathcal{F}$ Surg 1973;60:213-15.

3 Williamson RCN. Acalculous disease of the gall bladder. Gut 1988;29:86072.

4 Kang JY, Williamson RCN. Cholecystitis without gallstones. HPB Surg $1990 ; 2: 83-103$.

5 Nathan MH, Newman A, Murray DJ, et al. Cholecystokinin cholecystography. AfR Am ₹ Roentgenol 1970;110:240-51.

6 Fink-Bennett D, DeRidder P, Kolozsi WZ, et al. Cholecystokinin cholescintigraphy: Detection of abnormal gallbladder motor function in patients with chronic acalculous gallbladder disease. $f$ Nucl Med 1991;32:1695-9.

7 Misra DC, Blossom GB, Fink-Bennett D, et al. Results of surgical therapy for biliary dyskinesia. Arch Surg 1991;126:957-60.

8 Byrne P, Hunter GJS, Vallon A. Cholecystokinin cholecystography: a three year prospective trial. Clin Radiol 1985;36:499-502.

9 Rhodes M, Lennard TWJ, Farndon JR, et al. Cholecystokinin (CCK) provocation test: long-term follow-up after cholecystectomy. $\mathrm{Br} \mathcal{F}$ Surg 1988;75:951-3. 